\title{
Statistical dynamics of dislocation systems: The influence of dislocation-dislocation correlations
}

\author{
M. Zaiser \\ Max-Planck-Institut für Metallforschung, D-70569 Stuttgart, Germany \\ M.-Carmen Miguel \\ University of Barcelona, Dept. de Fisica Fonamental, Av. Diagonal 647, 08028 Barcelona, Spain \\ I. Groma \\ Eötvös University, Department of General Physics, P.O. Box 32, 1518 Budapest, Hungary
}

(Received 26 January 2001; published 19 November 2001)

\begin{abstract}
During plastic deformation of crystalline materials, the collective dynamics of interacting dislocations gives rise to various patterning phenomena. A crucial and still open question is whether the long range dislocationdislocation interactions which do not have an intrinsic range can lead to spatial patterns which may exhibit well-defined characteristic scales. It is demonstrated for a general model of two-dimensional dislocation systems that spontaneously emerging dislocation pair correlations introduce a length scale which is proportional to the mean dislocation spacing. General properties of the pair correlation functions are derived, and explicit calculations are performed for a simple special case, viz pair correlations in single-glide dislocation dynamics. It is shown that in this case the dislocation system exhibits a patterning instability leading to the formation of walls normal to the glide plane. The results are discussed in terms of their general implications for dislocation patterning.
\end{abstract}

DOI: 10.1103/PhysRevB.64.224102

PACS number(s): 62.20.Fe, 61.72.Lk, 05.90.+m

\section{INTRODUCTION}

In plastically deforming crystals, the accumulation, interaction and motion of large numbers of dislocations gives rise to a complex spatiotemporal dynamics. In many cases the dislocations form spatial patterns with length scales which decrease in inverse proportion with the flow stress ("law of similitude"). ${ }^{1}$ In conjunction with the Taylor relationship according to which the flow stress of a dislocation arrangement is proportional to the square root of dislocation density, this implies that the characteristic scales of deformation-induced dislocation patterns are often proportional to, but in general much larger than, the mean dislocation spacing. This holds even for "fractal" dislocation patterns which are self-similar over a certain range of scales, since the upper and lower bounds of this scaling regime are again proportional to the average dislocation spacing. ${ }^{2}$

In the past different phenomenological models have been proposed for dislocation patterning. While these models differ with respect to the conceptual framework employed and the way how length scales are introduced, they have in common that the dislocation arrangement is characterized in terms of space-dependent dislocation densities for which balance equations are formulated in a phenomenological manner. In the work of Walgraef and Aifantis, ${ }^{3,4}$ the framework of reaction-diffusion systems was used, and space dependencies were introduced through second-order gradient terms in the dislocation densities. Kratochvil proposed to describe spatial interactions in terms of nonlocal expressions either for the flow stress evolution in general ("nonlocal hardening") ${ }^{5}$ or, more specifically, for the sweeping of edge dislocation dipoles by moving screw dislocations. ${ }^{6}$ In one of the earliest papers on the subject, Holt ${ }^{7}$ used an irreversible thermodynamics framework and related the formation of or- dered patterns to the minimization of elastic energy. In this approach dislocation pair correlations had to be introduced phenomenologically in order to obtain a well-defined energy functional. ${ }^{7,8}$ The pattern wavelength obtained by Holt turned out to be proportional to the spatial range of the correlations.

All these approaches have in common that the wavelength of dislocation patterns deduced from the models is governed by length scales introduced a priori in terms of gradient coefficients, nonlocal kernels or correlation functions. Since the results are predetermined by the phenomenological "input," it is arguable whether this kind of models can solve the problem of length scale selection. This deficiency has led to the idea of using stochastic models ${ }^{9,10}$ where dislocation interactions are considered in terms of spatiotemporal fluctuations of the local dislocation fluxes (shear strain rates). This approach yields a characterization of inhomogeneous dislocation arrangements in terms of distributions of dislocation densities which give a statistical "signature" of patterning but no direct information on how the dislocations are distributed in space. The approach does not solve the problem of length scale selection but has been fruitful in modeling multiscale dislocation patterns such as fractal cell structures observed in deformation of high-symmetry oriented fcc crystals. ${ }^{11-14}$ Furthermore, it has been demonstrated that the information contained in the dislocation density probability distribution is sufficient to calculate important properties such as the macroscopic flow stress of inhomogeneous dislocation arrangements ${ }^{2,15}$ and characteristics of the x-ray line profiles. ${ }^{14}$

In addition to the theoretical models mentioned above, over the past few years a vast number of computer simulations have been performed for studying the dislocation patterning phenomenon. Using different discretization methods, 
several authors (see, e.g., Refs. 16,17) have investigated the evolution of systems of interacting dislocation lines. In these works [often referred to as three-dimensional (3D) simulations] the dislocations are treated as real linear entities. While early stages of patterning have been observed in such simulations, ${ }^{18,19}$ statistically reliable information on the mechanisms which govern the pattern wavelengths cannot yet be deduced from the simulations since the characteristic lengths of the observed rudimentary patterns are of the same order as the size of the simulation volume. Hence, such 3D simulations provide insight into often complex but still "elementary" dislocation phenomena. The results of these works are very important but the study of dislocation patterning requires approaching the problem on a scale larger than that one can presently afford with the computation power available. A computationally less demanding approach is to investigate numerically the collective behavior of an ensemble of parallel straight dislocations. Certainly, this is a strong simplification of a real dislocation network but, on the other hand, such a 2D system is an ideal model system for investigating the influence of long range dislocationdislocation interactions. Since the precise roles of the short range (junction formation) and long range dislocation interactions in dislocation patterning are not clear at the moment, it is an important question whether the long range interactions alone (which decay as $1 / r$ and hence do not have an intrinsic range) can give rise to dislocation arrangements with well-defined length scales. The objective of the present paper is to analyze the consequences of the long range dislocation-dislocation interactions in a 2D setting within an analytical framework, with a special attention on the properties of dislocation-dislocation correlation functions.

To characterize the dynamics of a 2D dislocation system, we adopt the statistical approach proposed by Groma, ${ }^{20}$ which is based on many-dislocation densities. Before going into the technicalities, we find it useful to state the main problem in statistical modelling of dislocation systems in physical terms: On "mesoscopic" scales large as compared to the dislocation spacing, the dislocation arrangement can be characterized by single-dislocation densities which represent probabilities to find dislocations of a given type at a given point in space. The average Kröner-Nye dislocation density tensor can be computed from these single-dislocation densities, see the work of El-Azab ${ }^{21}$ where this is demonstrated for three-dimensional dislocation arrangements. From this tensor, in turn, long-range stresses in the dislocation arrangement can be calculated using the stress function method. ${ }^{22,23}$ However, it has been recognized already in an early paper by Kröner ${ }^{24}$ that the information contained in spatially averaged single-dislocation densities and the corresponding mesoscopically averaged internal stress fields is not sufficient to statistically characterize the local internal stress state on the microscopic scale of individual dislocations. This, however, is indispensable for studying the dynamics of dislocation systems: Motion of dislocations takes place under the influence of the locally acting external and internal stresses. The internal stress experienced by a given dislocation depends on the configuration of the other dislocations in its surrounding. This configuration is obviously influenced by the presence of the first one- a fact which cannot be grasped by spatially averaged densities which represent probabilities for the spatial occurrence of a given type of dislocations irrespective of their arrangement relative to each other. Hence one has to ask for conditional probabilities to find other dislocations in a certain position relative to the dislocation under consideration, which mathematically implies the consideration of pair densities or pair correlations. The evolution of pair configurations depends, in turn, on the arrangement of third dislocations, and so forth.

Therefore it is necessary to consider in a systematic manner many-dislocation densities which characterize the probability of occurrence of many-dislocation configurations. A formal procedure for defining such densities and obtaining the corresponding equations of evolution from the dynamics of the discrete dislocations has been discussed in an earlier work by Groma. ${ }^{20}$ For three-dimensional dislocation systems, a systematic method to obtain a statistical description has been demonstrated by El-Azab. ${ }^{21}$ El-Azab uses a conceptual framework which differs from that used by Groma and is similar to Klimontovic's approach in nonequilibrium statistical mechanics. ${ }^{25}$ His approach is of particular interest since it permits in principle the characterization of correlations in 3D dislocation systems. For 2D systems, however, the results derived by El-Azab are equivalent to those obtained by Groma. Both Groma and El-Azab use mean-field approximations which reduce the problem to single-dislocation densities. ${ }^{20,26,27,21}$ A stochastic generalization of Groma's model which takes into account microscopic stress fluctuations in terms of a random stochastic process was shown to yield fractal dislocation patterns. ${ }^{27}$ However, these studies also demonstrated that the neglection of correlations which is inherent in any mean-field approach makes it impossible to solve the problem of length scale selection. In the present investigation, we therefore consider explicitly the evolution of correlations in the dislocation arrangement. In Sec. II, the problem is stated in mathematical terms and a scaling analysis is performed. In the rate-independent case, the scaling behavior of the evolution equations is found to be consistent with the "law of similitude" and the Taylor relationship. In Secs. III and IV, general properties of pair correlation functions are derived and compared with simulation results for the case where dislocations of only one slip system are present. The role of pair correlations in dislocation patterning is discussed in Sec. V, and general conclusions are given in Sec. VI.

\section{STATISTICAL CHARACTERIZATION OF A 2-DIMENSIONAL DISLOCATION ARRANGEMENT}

\section{A. Mathematical formulation}

We consider an arrangement of $N$ dislocations with line direction parallel to the $z$ axis of a Cartesian coordinate system and denote by $\boldsymbol{r}_{i}(i=1 \ldots N)$ the position vectors of the dislocations in the $x y$ plane. In generalization of the situation discussed in Ref. 20, dislocation activity is allowed in more than one slip system. However, because of the 2D geometry considered, all allowed glide directions are contained in the $x y$ plane. The slip system of the $i$ th dislocation (Burgers 
vector and glide direction) is labeled by $\alpha_{i}$, and the sign of this dislocation by $s_{i}\left(s_{i}= \pm 1\right)$. For simplicity, the dislocation strength $b$ (the modulus of the Burgers vector) is assumed to be the same for all dislocations. During plastic deformation, due to dislocation multiplication or annihilation the number of dislocations is not conserved. In principle, terms characterizing dislocation multiplication and dislocation reactions can be introduced into the present formalism. (For systems of reacting particles, this is, e.g., discussed in Ref. 29). However, in the present investigation we focus on the consequences of long-range interactions. Hence, for simplicity $N$ is kept constant.

Dislocation glide is governed by the component of the Peach-Koehler force ${ }^{30}$ in the glide direction. For the $i$ th dislocation this is given by $F_{i}\left(\boldsymbol{r}_{i}\right)=s_{i} b \sigma^{\alpha_{i}}\left(\boldsymbol{r}_{i}\right)$, where $\sigma^{\alpha_{i}}\left(\boldsymbol{r}_{i}\right)$ is the resolved shear stress in the slip system $\alpha_{i}$ at the position of this dislocation. We assume overdamped dislocation motion and a friction force which is linearly proportional to the dislocation velocity $\boldsymbol{v}_{i}=\boldsymbol{e}^{\alpha_{i}} \mu F_{i}\left(\boldsymbol{r}_{i}\right)$, where $\mu$ is an effective mobility coefficient and $\boldsymbol{e}^{\alpha_{i}}$ is the unit vector in the glide direction of dislocations of slip system $\alpha_{i}$ (the line of intersection between the glide plane and the $x y$ plane). Taking into account that the local shear stress acting on the dislocation is the sum of the external shear stress and the shear stresses created by all other dislocations, the velocity of the $i$ th dislocation may be written as

$$
\boldsymbol{v}_{i}=s_{i} \mu b \boldsymbol{e}^{\alpha_{i}}\left[\sigma^{\mathrm{ext}, \alpha_{i}}+\sum_{k \neq i}^{N} s_{k} \boldsymbol{\sigma}^{\alpha_{i} \alpha_{k}}\left(\boldsymbol{r}_{i}-\boldsymbol{r}_{k}\right)\right] .
$$

Here $\sigma^{\text {ext, } \alpha_{i}}$ is the external stress resolved in the slip system $\alpha_{i}$ and $\sigma^{\alpha_{i} \alpha_{k}}\left(\boldsymbol{r}_{i}-\boldsymbol{r}_{k}\right)$ is the shear stress produced in slip system $\alpha_{i}$ at the position $\boldsymbol{r}_{i}$ by a (positive) dislocation of slip system $\alpha_{k}$ located at $\boldsymbol{r}_{k}$. These stresses are related via

$$
\sigma^{\mathrm{ext}, \alpha}=\sum_{i j} \sigma_{i j}^{\mathrm{ext}} M_{i j}^{\alpha}, \quad \sigma^{\alpha \alpha^{\prime}}(\boldsymbol{r})=\sum_{i j} \sigma_{i j}^{\alpha^{\prime}}(\boldsymbol{r}) M_{i j}^{\alpha}
$$

to the external stress field and the stress field of a single dislocation, respectively. The projection tensors $M_{i j}^{\alpha}$ are given by $M_{i j}^{\alpha}=(1 / 2 b)\left[b_{i}^{\alpha} n_{j}^{\alpha}+n_{i}^{\alpha} b_{j}^{\alpha}\right]$ where $b_{i}^{\alpha}$ and $n_{j}^{\alpha}$ are the components of the Burgers vector $\boldsymbol{b}^{\alpha}$ and the glide plane normal $\boldsymbol{n}^{\alpha}$ of slip system $\alpha$. The dislocation stress fields $\sigma_{i j}^{\alpha}(\boldsymbol{r})$ can be written as

$$
\sigma_{i j}^{\alpha}(\boldsymbol{r})=G b s_{i j}^{\alpha}(\boldsymbol{r})=G b \frac{K_{i j}^{\alpha}(\theta)}{|\boldsymbol{r}|}
$$

where $G$ is a shear modulus of the material and $K_{i j}^{\alpha}$ is a dimensionless function of the angle $\theta$ in the $x y$ plane. According to Eqs. (2) and (3), the resolved shear stresses $\sigma^{\alpha_{k} \alpha_{l}}(\boldsymbol{r})=G b K^{\alpha_{k} \alpha_{l}}(\theta) /|\boldsymbol{r}|$, where $K^{\alpha_{k} \alpha_{l}}=\Sigma_{i j} M_{i j}^{\alpha_{k}} K_{i j}^{\alpha_{l}}$.

In principle, the evolution of the dislocation arrangement can be obtained by tracing the individual dislocation positions using Eq. (1). However, the plastic behavior of a deforming crystal depends on statistical properties of the dislocation ensemble rather than on the precise location of the individual dislocations. We consider an ensemble of statistically equivalent dislocation arrangements and define manydislocation densities by the ensemble averages

$$
\begin{aligned}
\rho_{(1)}^{\alpha, s}(\boldsymbol{r}) & :=\left\langle\sum_{k=1}^{N} \delta_{\alpha \alpha_{k}} \delta_{s s_{k}} \delta\left(\boldsymbol{r}-\boldsymbol{r}_{k}\right)\right\rangle, \\
\rho_{(2)}^{\alpha \alpha^{\prime}, s s^{\prime}}\left(\boldsymbol{r}, \boldsymbol{r}^{\prime}\right):= & \left\langle\sum_{k=1}^{N} \sum_{l \neq k}^{N} \delta_{\alpha \alpha_{k}} \delta_{\alpha^{\prime} \alpha_{l}} \delta_{s s_{k}} \delta_{s^{\prime} s_{l}} \delta\left(\boldsymbol{r}-\boldsymbol{r}_{k}\right)\right. \\
& \left.\times \delta\left(\boldsymbol{r}^{\prime}-\boldsymbol{r}_{l}\right)\right\rangle, \ldots
\end{aligned}
$$

In Eq. (4), $\rho_{(1)}^{\alpha, s}(\boldsymbol{r})$ is the density of dislocations of type $\{\alpha, s\}$ at the position $\boldsymbol{r}$, and $\rho_{(2)}^{\alpha \alpha^{\prime}, s s^{\prime}}\left(\boldsymbol{r}, \boldsymbol{r}^{\prime}\right)$ is the density of pairs of dislocations of types $\{\alpha, s\}$ and $\left\{\alpha^{\prime}, s^{\prime}\right\}$ at the respective positions $\boldsymbol{r}$ and $\boldsymbol{r}^{\prime}$. Higher-order densities which obey the recursive relations

$$
\rho_{(n)}^{\alpha_{1} \cdots \alpha_{n}, s_{1} \cdots s_{n}}\left(\boldsymbol{r}_{1} \cdots \boldsymbol{r}_{n}\right)=\frac{1}{N-n} \sum_{\alpha_{n+1} s_{n+1}} \int \rho_{(n+1)}^{\alpha_{1} \cdots \alpha_{n+1}, s_{1} \cdots s_{n+1}}\left(\boldsymbol{r}_{1} \cdots \boldsymbol{r}_{n+1}\right) d^{2} r_{n+1}
$$

are defined accordingly. It is noted that owing to the ensemble averaging the many-dislocation densities are in general smooth functions of their arguments. In the following, we shall often use an abbreviated notation, writing $\rho_{(1)}(1):=\rho_{(1)}^{\beta_{1}, s_{1}}\left(\boldsymbol{r}_{1}\right)$, $\rho_{(2)}(1,2):=\rho_{(2)}^{\beta_{1} \beta_{2}, s_{1} s_{2}}\left(\boldsymbol{r}_{1}, \boldsymbol{r}_{2}\right)$, etc.

To formulate the evolution of the many-dislocation densities, we ensemble average the discrete equations of motion (1). Using the definitions given in Eq. (4) we obtain for the evolution of the single-dislocation densities $\rho_{(1)}^{\alpha, s}$ (see Ref. 20)

$$
\frac{1}{b \mu} \partial_{t} \rho_{(1)}(1)=-\nabla_{1}^{\alpha_{1}} s_{1} \sigma^{\mathrm{ext}, \alpha_{1}} \rho_{(1)}(1)-\nabla_{1}^{\alpha_{1}} \sum_{\alpha_{2}, s_{2}} s_{1} s_{2} \int \rho_{(2)}(1,2) \sigma^{\alpha_{1} \alpha_{2}}\left(\boldsymbol{r}_{1}-\boldsymbol{r}_{2}\right) d^{2} r_{2},
$$

i.e., the equations of evolution of the single-dislocation densities contain integrals over the pair densities. The operator $\nabla_{i}^{\alpha_{i}}$ $=\vec{\nabla}_{\boldsymbol{r}_{i}} \boldsymbol{e}^{\alpha_{i}}$ acts on the coordinate $\boldsymbol{r}_{i}$ and takes the directional derivative in the direction $\boldsymbol{e}^{\alpha_{i}}$.

It may be mentioned that the right-hand side of this equation is the gradient of the dislocation flux $\boldsymbol{j}^{\alpha_{1}, s_{1}}$ of dislocations of slip system $\alpha_{1}$ and sign $s_{1}$, divided by $b \mu$. From the dislocation fluxes, the components of the plastic strain rate tensor are obtained as (see Ref. 28) 


$$
\dot{\varepsilon}_{k l}=b \sum_{\alpha, s} s\left(\dot{j}^{\alpha, s} \boldsymbol{e}^{\alpha}\right) M_{k l}^{\alpha} .
$$

Furthermore, by multiplying Eq. (6) with $s_{1} \boldsymbol{b}^{\alpha_{1}} \boldsymbol{n}^{\alpha_{1}}$ and summing over all $\alpha_{1}$ and $s_{1}$, one can arrive at an equation having the form

$$
\frac{\partial \boldsymbol{\alpha}}{\partial t}+\operatorname{curl} \boldsymbol{J}=0
$$

in which $\boldsymbol{\alpha}$ is Kröner's dislocation density tensor ${ }^{22}$ and $\boldsymbol{J}$ is the dislocation current density tensor, the symmetric part of which is again the plastic strain rate. It follows, that from Eq. (6) we do have explicit expressions for the plastic strain rate, which build the link between the formalism presented and macroscopic deformation problems.

The pair densities obey, in turn, the equations

$$
\frac{1}{b \mu} \partial_{t} \rho_{(2)}(1,2)=-\nabla_{1}^{\alpha_{1}} s_{1}\left[\sigma^{\mathrm{ext}, \alpha_{1}}+s_{2} \sigma^{\alpha_{1} \alpha_{2}}\left(\boldsymbol{r}_{1}-\boldsymbol{r}_{2}\right)\right] \rho_{(2)}(1,2)-\nabla_{1}^{\alpha_{1}} \sum_{\alpha_{3}, s_{3}} s_{1} s_{3} \int \rho_{(3)}(1,2,3) \sigma^{\alpha_{1} \alpha_{3}}\left(\boldsymbol{r}_{1}-\boldsymbol{r}_{3}\right) d^{2} r_{3}+1 \leftrightarrow 2
$$

Here the symbol $1 \leftrightarrow 2$ means that the terms on the right-hand side of Eq. (9) are repeated with indices 1 and 2 interchanged. The general equation for the $n$-dislocation density is

$$
\begin{aligned}
& \frac{1}{b \mu} \partial_{t} \rho_{(n)}(1 \cdots n)=-\sum_{j=1}^{n} \nabla_{j}^{\alpha_{j}} s_{j}\left[\sigma^{\mathrm{ext}, \alpha_{j}}+\sum_{k \neq j}^{n} s_{k} \sigma^{\alpha_{j} \alpha_{k}\left(\boldsymbol{r}_{j}-\boldsymbol{r}_{k}\right)}\right] \boldsymbol{\rho}_{(n)}(1 \cdots n)-\sum_{j=1}^{n} \nabla_{j}^{\alpha_{j}} \sum_{\alpha_{n+1}, s_{n+1}} s_{j} s_{n+1}
\end{aligned}
$$

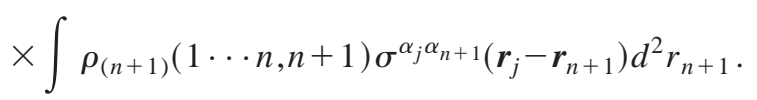

Knowledge of the dislocation densities of arbitrary order yields a complete statistical description of the dislocation system, i.e., the statistical information obtained by solving the infinite hierarchy of equations $(6),(9),(10)$ is equivalent to the information obtained by solving the discrete equations of motion for infinitely many statistically equivalent initial configurations and subsequent averaging. However, the hierarchy of Eqs. (6),(9),(10) where the equations for the $n$-dislocation densities contain integrals over the densities of next-higher order is much more difficult to handle than the discrete equations. Therefore, the statistical approach used in the present work is useful only if relevant information can be obtained by truncating this hierarchy at some low order. To find out at which order truncation may be done without destroying essential qualitative features of the dynamics, it is useful to study some scaling properties of the full system of Eqs. (6),(9),(10).

\section{B. Scaling relations}

We investigate the scaling behavior of Eqs. (6),(9),(10) by assuming that all single-dislocation densities are changed by a common factor $C_{\rho}, \rho_{(1)}^{\alpha, s} \rightarrow C_{\rho} \rho_{(1)}^{\alpha, s}$. Further we require that the $n$-dislocation densities scale like products of $n$ singledislocation densities. Now we look for an appropriate transformation of the other quantities in Eqs. (6),(9),(10) such that these equations remain invariant upon the rescaling. Power counting shows that there is exactly one transformation which fulfills this requirement; it is given by

$$
\begin{gathered}
\rho_{(1)}^{\alpha, s} \rightarrow C_{\rho} \rho_{(1)}^{\alpha, s}, \quad \rho_{(n)}^{\alpha_{1} \cdots \alpha_{n}, s_{1} \cdots s_{n}} \rightarrow C_{\rho}^{n} \rho_{(n)}^{\alpha_{1} \cdots \alpha_{n}, s_{1} \cdots s_{n}}, \\
\boldsymbol{r} \rightarrow C_{\rho}^{-1 / 2} r, \quad t \rightarrow C_{\rho}^{-1} t, \quad \sigma_{i j}^{\mathrm{ext}} \rightarrow C_{\rho}^{1 / 2} \sigma_{i j}^{\mathrm{ext}} .
\end{gathered}
$$

Invariance under this transformation implies that any particular solution of $(6),(9),(10)$ belongs to a one-parameter manifold of similitude solutions pertaining to different external stresses. These solutions may be parametrized, for instance, by their total dislocation density $\rho:=[1 / V] \Sigma_{\alpha, s} \int_{V} \rho_{(1)}^{\alpha, s}(\boldsymbol{r}) d^{2} r$ where $V$ is the crystal volume. From a given solution, the corresponding similitude manifold is generated by scaling all lengths in proportion with the mean dislocation spacing $1 / \sqrt{\rho}$, all times in proportion with $1 / \rho$, and the externally applied stress in proportion with $\sqrt{\rho}$.

When the dislocation mobilities are high, dislocations almost instantaneously relax into (quasi)stationary configurations. In the limit of rate-independent behavior $(\mu \rightarrow \infty)$ where dislocations respond instantaneously to acting stresses, the dislocations are always arranged in metastable stationary configurations, the lifetime of which is governed by the activation of new sources or an increase of the external stress. In the quasistatic limit, the scaling behavior of Eqs. (6),(9),(10) is consistent with two basic empirical properties of dislocation systems. (i) According to the Taylor relationship, the flow stress (i.e., the stress at which large-scale dislocation motion sets in) scales as the square root of dislocation density. (ii) According to the "law of similitude" the length scales of dislocation patterns observed after plastic deformation scale in inverse proportion with the flow stress 
(and hence in proportion with the mean dislocation spacing). In the course of strain hardening, dislocation arrangements are often found to evolve on a similitude manifold where they contract in space but maintain their geometrical characteristics. ${ }^{31}$

\section{PAIR CORRELATIONS IN QUASISTATIC DISLOCATION ARRANGEMENTS: GENERAL PROPERTIES}

The scaling property (11) gives an important guideline how to handle the infinite hierarchy of many-dislocation densities. The idea is to truncate this hierarchy at some level $n$ by expressing densities of order $n+1$ in terms of densities of lower order. The simplest manner to do this is to use, in Eq. (6), the mean-field approximation $\rho_{(2)}(1,2) \approx \rho_{(1)}(1) \rho_{(1)}(2)$ as proposed by Groma. ${ }^{20}$ This amounts to the assumption that the positions of individual dislocations are uncorrelated. However, when we require that truncation preserves the fundamental scaling properties expressed by Eq. (11), it becomes obvious that the mean-field approximation which truncates at first order destroys substantial information: The length scale contained in the full hierarchy - the mean dislocation spacing - no longer shows up in the mean-field equation, which therefore permits additional scaling transformations. On the other hand, Eq. (9), which involves pair densities, has the same scaling properties as the general Eq. (10). Hence, consideration of pair densities (or, equivalently, pair correlations) allows one to account for all scaling relations between stress, total dislocation density, and length scales of the dislocation pattern.

We focus on weakly correlated dislocation arrangements where distant dislocations behave in a statistically independent manner. Then the pair densities fulfill the asymptotic relations

$$
\begin{gathered}
\rho_{(2)}(1,2) \rightarrow \rho_{(1)}(1) \rho_{(2)}(2) \\
\text { for } r_{12}:=\left|\boldsymbol{r}_{1}-\boldsymbol{r}_{2}\right| \rightarrow \infty
\end{gathered}
$$

For the third-order densities the required asymptotic behavior is

$$
\begin{gathered}
\rho_{(3)}(1,2,3) \rightarrow \rho_{(2)}(1,2) \rho_{(1)}(3) \\
\text { for } r_{13} \rightarrow \infty \text { and } r_{23} \rightarrow \infty
\end{gathered}
$$

To truncate the hierarchy of many-dislocation densities at second order in a manner which is consistent with Eqs. (11) and (12), we use Kirkwood's approximation

$$
\rho_{(3)}(1,2,3) \approx \frac{\rho_{(2)}(1,2) \rho_{(2)}(2,3) \rho_{(2)}(3,1)}{\rho_{(1)}(1) \rho_{(1)}(2) \rho_{(1)}(3)} .
$$

In the quasistatic case where time derivatives may be neglected, we obtain from Eqs. (6) and (9) in this approximation the integral equation

$$
\begin{aligned}
s_{2} \sigma^{\alpha_{1} \alpha_{2}}\left(\boldsymbol{r}_{1}-\boldsymbol{r}_{2}\right)= & \rho \sum_{\alpha_{3}, s_{3}} s_{3} \int f^{\alpha_{3} s_{3}}\left(\boldsymbol{r}_{3}\right) d_{(23)}\left(\boldsymbol{r}_{2}, \boldsymbol{r}_{3}\right) \\
& \times\left[1+d_{(31)}\left(\boldsymbol{r}_{3}, \boldsymbol{r}_{1}\right)\right] \sigma^{\alpha_{1} \alpha_{3}}\left(\boldsymbol{r}_{1}-\boldsymbol{r}_{3}\right) d^{2} r_{3},
\end{aligned}
$$

where $\quad f^{\alpha, s}(\boldsymbol{r}):=\rho_{(1)}^{\alpha, s}(\boldsymbol{r}) / \rho, \quad$ and $\quad d_{(12)}\left(\boldsymbol{r}_{1}, \boldsymbol{r}_{2}\right):=$ $\rho_{(2)}(1,2) /\left[\rho_{(1)}(1) \rho_{(1)}(2)\right]-1$ is the scaled pair correlation function of dislocations of types $\left\{\alpha_{1}, s_{1}\right\}$ and $\left\{\alpha_{2}, s_{2}\right\}$. To study the properties of these pair correlation functions, we consider the case of a homogeneous dislocation arrangement where $f^{\alpha, s}=\operatorname{const}(\boldsymbol{r})$ for all $\{\alpha, s\}$. Then the $d_{(12)}$ are functions of the relative positions $\boldsymbol{r}_{1}-\boldsymbol{r}_{2}$ only, and some general properties of these functions can be obtained from simple inspection of Eq. (15), considering the behavior at large and small values of $r_{12}$.

The pair correlation functions depend only on the scaled space coordinates $\tilde{\boldsymbol{r}}:=\boldsymbol{r} \sqrt{\rho}$. This reflects the scaling behavior discussed above.

At small $\tilde{r}_{12}$, the pair correlation functions must exhibit a $1 / \tilde{r}_{12}$ singularity.

The integrals $\hat{d}_{(12)}:=\int d_{(12)}(\tilde{\boldsymbol{r}}) d^{2} \tilde{r}$ must be finite. In Fourier space at $k=0$ all derivatives of the Fourier transforms of the $d_{(12)}$ and of the $\left[d_{(12)} \sigma^{\alpha_{1} \alpha_{2}}\right]$ must also be finite, which implies that in real space the $d_{(12)}$ decay faster than algebraically.

For large $\tilde{r}_{12}$ one may therefore approximate $d_{(12)}\left(\tilde{r}_{12}\right)$ $\approx \hat{d}_{(12)} \delta\left(\tilde{\boldsymbol{r}}_{12}\right)$. Equation (15) in this approximation reduces to

$$
s_{2} \sigma^{\alpha_{1} \alpha_{2}}\left(\boldsymbol{r}_{12}\right)=-\sum_{\alpha_{3} s_{3}} s_{3} f^{\alpha_{3} s_{3}} \hat{d}_{(23)} \sigma^{\alpha_{1} \alpha_{3}}\left(\boldsymbol{r}_{12}\right)
$$

This equation must be fulfilled by the "amplitudes" $\hat{d}_{(23)}$ of the pair correlation functions for all combinations $\left\{\alpha_{1} \ldots \alpha_{3}, s_{2}, s_{3}\right\}$ and irrespective of the direction of the vector $\boldsymbol{r}_{12}$.

To interpret Eq. (16) in physical terms, we note that [1 $\left.+d_{(12)}\left(\boldsymbol{r}_{12}\right)\right] \rho_{(1)}^{\alpha_{2} s_{2}} d V$ is the conditional probability to find a dislocation of type 2 in a volume element $d V$ at $\boldsymbol{r}_{2}$ when there is a dislocation of type 1 at $\boldsymbol{r}_{1}$. For a homogeneous random arrangement of dislocations, this probability reduces to $\rho_{(1)}(2) d V$. Accordingly, $d_{(12)}\left(\boldsymbol{r}_{12}\right) \rho_{(1)}(2)$ can be understood as an average density and $\hat{d}_{(12)}\left(\boldsymbol{r}_{1}\right) f^{\alpha_{2} s_{2}}$ as the total number of formal "excess dislocations" of type 2 surrounding a dislocation of type 1 in a correlated arrangement. Anticorrelations are formally represented by negative excess dislocations. Equation (16) simply means that the shear stresses created by a dislocation of type 2 in any slip system $\alpha_{1}$ are, at large distances and in all directions, balanced by the stresses of the surrounding excess dislocations. This "screening condition" ensures that the energy density of the elastic field is finite in spite of a diverging self-energy of the isolated dislocations; an equivalent formulation of Eq. (16) is that the sum of the Burgers vectors of the formal "excess dislocations" and the Burgers vector of the first dislocation is zero. $^{32}$ 


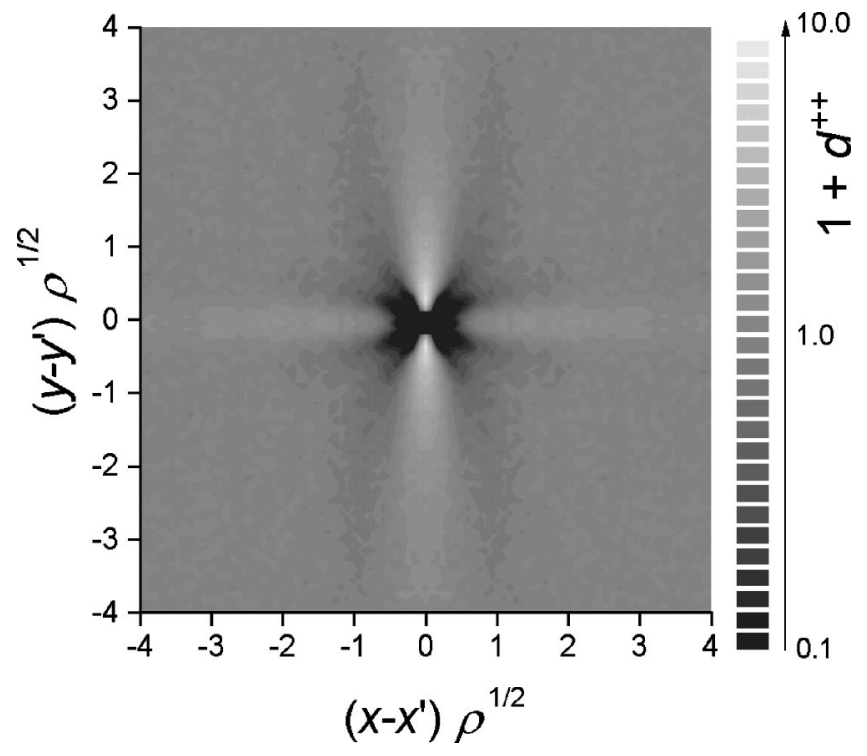

FIG. 1. Correlation function of dislocations of the same sign in scaled coordinates, stress-free state; for details see text.

\section{PAIR CORRELATION FUNCTIONS FOR A SINGLE SLIP SYSTEM}

To assess the validity of the general results obtained in the previous section, we consider pair correlation functions obtained from simulations of the dynamics of systems of discrete edge dislocations moving in a single slip system. ${ }^{33}$ Accordingly, we drop the superscript $\alpha$ and distinguish dislocations only according to their sign $s \in\{+,-\}$. The external resolved shear stress in the slip system is denoted as $\sigma^{\text {ext }}$, the interaction stress (shear stress in the slip system) between two positive dislocations is $\sigma(\boldsymbol{r})$, and the glide direction is identified with the $x$ direction.

Figures 1 and 2 show pair correlation functions $d_{++}$and $d_{+-}$of dislocations of equal and of opposite signs. These

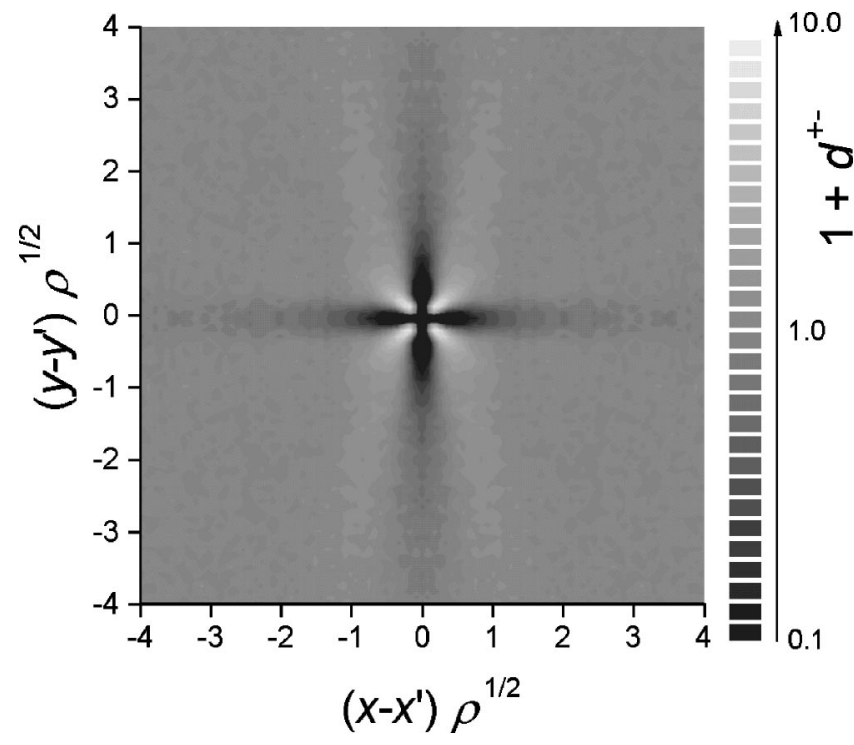

FIG. 2. Correlation function of dislocations of opposite signs; as Fig. 1.

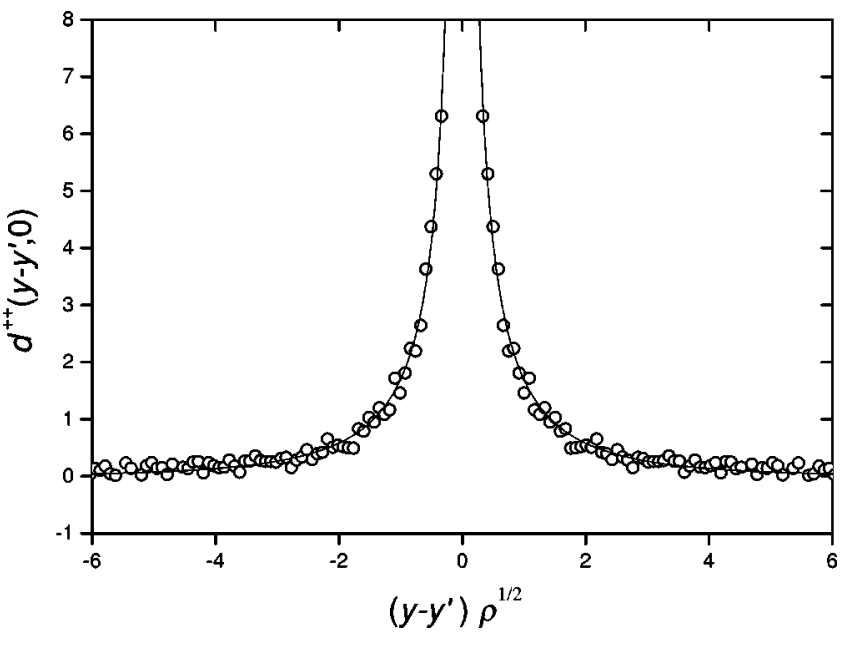

FIG. 3. Decay of the correlation function of dislocations of the same sign in the $y$ direction.

functions have been obtained from a simulation at zero external stress: Equal numbers of dislocations of both signs were initially placed at random, and pair densities were determined after relaxation of the dislocation system to a stationary configuration. Dislocations of opposite signs approaching each other at distances below the core radius $r_{\mathrm{c}}$ $\approx b$ were removed from the system. Figure 1 shows a tendency of dislocations of the same sign to form walls where they arrange perpendicularly above each other, while dislocations of opposite signs tend to form close dipoles with $45^{\circ}$ orientations (Fig. 2). The behavior of the pair correlation functions at distances of about one average dislocation spacing indicates a tendency of the dipoles to arrange vertically above each other. The behavior of the pair correlation functions obtained from the simulation is consistent with the results obtained in the previous sections: (i) at short distances the functions exhibit a $1 / r$ singularity (this is truncated at the core radius); (ii) At large distances, correlations decay exponentially. This is illustrated by Fig. 3 showing the pair correlation function $d_{++}$of dislocations of the same sign arranged vertically above each other as a function of the scaled coordinate $\tilde{y}:=y \sqrt{\rho}$ where $\rho=\rho_{(1)}^{+}+\rho_{(1)}^{-}$and the $y$ direction is normal to the slip plane. The data are well approximated by the fit function $f(\tilde{y}) \propto(1 / \tilde{y}) \exp [-0.38 \tilde{y}]$, i.e., the effective range of pair correlations is restricted to a few dislocation spacings.

It can be shown by symmetry arguments that, under zero stress, the pair correlation functions for a single slip system are invariant (i) with respect to a change of sign of both dislocations and (ii) with respect to the transformation $\boldsymbol{r} \rightarrow$ $\boldsymbol{- r}$. When an external stress is applied, these symmetries still hold for the correlation function of dislocations of the same sign. The simulations indicate that this function is practically unchanged unless the stresses are very high. For dislocations of opposite sign, however, the symmetries are broken and only invariance under the simultaneous inversion of signs and space coordinates of the dislocations is preserved, $d_{+-}(\boldsymbol{r})=d_{-+}(-\boldsymbol{r})$. Under applied external stress, the mutual arrangement of dislocations of opposite signs changes 


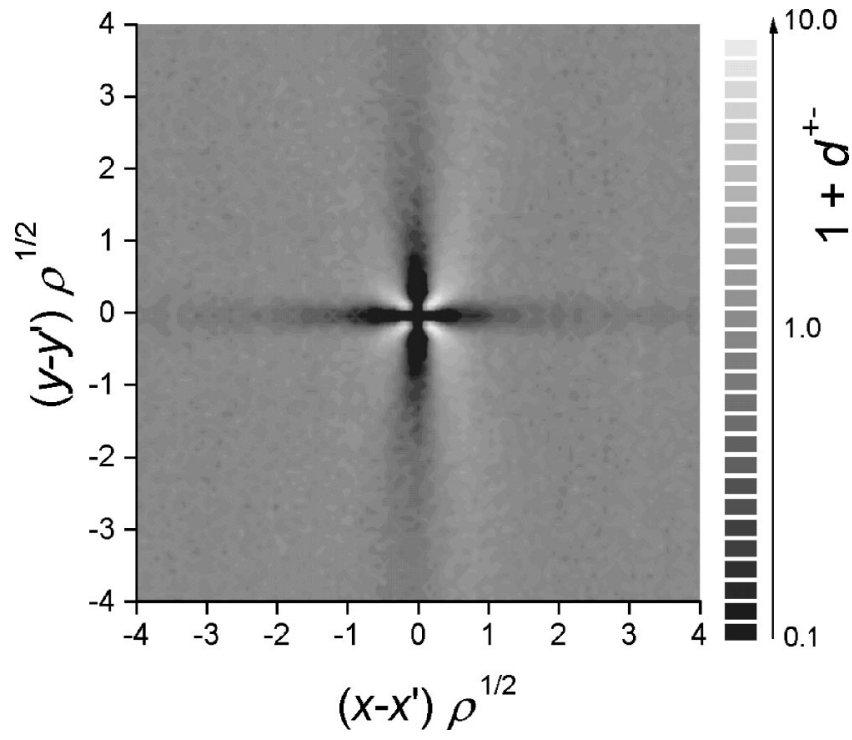

FIG. 4. Correlation function of dislocations of opposite signs in scaled coordinates, external stress $=0.15 /(G b \sqrt{\rho})$.

such that these dislocations exert a back stress on each other which offsets the externally applied stress. The corresponding "polarization" of the pair correlation function $d_{+-}$is seen in Fig. 4; the asymmetry of this function increases in proportion with the applied stress. In physical terms, this monitors the polarization of dislocation dipoles and multipoles.

\section{PAIR CORRELATIONS AND DISLOCATION PATTERNING}

The range of dislocation-dislocation correlations scales in proportion with the average dislocation spacing. Therefore, accounting for pair correlations introduces a characteristic length scale into the equations of evolution of the dislocation densities. To illustrate this, we consider again the simplest case, namely, an arrangement of equal numbers of edge dislocations of both signs of total density $\rho$, on one slip system and without external stress. In this case, the equations of evolution of the dislocation densities can be written as

$$
\begin{aligned}
\frac{1}{b \mu} \partial_{t} \rho(\boldsymbol{r})= & -\nabla_{x} \int\left\{\phi(\boldsymbol{r}) \phi\left(\boldsymbol{r}^{\prime}\right)\left[1+d_{\phi}\left(\boldsymbol{r}-\boldsymbol{r}^{\prime}\right)\right]\right. \\
& \left.+\rho(\boldsymbol{r}) \rho\left(\boldsymbol{r}^{\prime}\right) d_{\rho}\left(\boldsymbol{r}-\boldsymbol{r}^{\prime}\right)\right\} \sigma\left(\boldsymbol{r}-\boldsymbol{r}^{\prime}\right) d^{2} r^{\prime} \\
\frac{1}{b \mu} \partial_{t} \phi(\boldsymbol{r})= & -\nabla_{x} \int\left\{\rho(\boldsymbol{r}) \phi\left(\boldsymbol{r}^{\prime}\right)\left[1+d_{\phi}\left(\boldsymbol{r}-\boldsymbol{r}^{\prime}\right)\right]\right. \\
& \left.+\phi(\boldsymbol{r}) \rho\left(\boldsymbol{r}^{\prime}\right) d_{\rho}\left(\boldsymbol{r}-\boldsymbol{r}^{\prime}\right)\right\} \sigma\left(\boldsymbol{r}-\boldsymbol{r}^{\prime}\right) d^{2} r^{\prime}
\end{aligned}
$$

Here $\rho(\boldsymbol{r})=\rho_{(1)}^{+}(\boldsymbol{r})+\rho_{(1)}^{-}(\boldsymbol{r})$ is the local dislocation density, $\phi:=\rho_{(1)}^{+}(\boldsymbol{r})-\rho_{(1)}^{-}(\boldsymbol{r})$ the local surplus dislocation density, and the effective pair correlation functions are given by $d_{\phi}$ $=[1 / 2]\left(d_{++}+d_{+-}\right)$and $d_{\rho}=[1 / 2]\left(d_{++}-d_{+-}\right)$.
We now study the linear stability of an initially homogeneous dislocation arrangement, $\rho(\boldsymbol{r})=\rho=$ const, $\phi(\boldsymbol{r})=0$, with respect to small space- and time-dependent perturbations. We consider the special case where the polarization $\phi=\rho_{(1)}^{+}-\rho_{(1)}^{-}$of the dislocation arrangement remains zero. (A more detailed analysis including effects of polarization and nonzero external stress as well as nonlinear effects will be published elsewhere.) Under these restrictions, the equation of evolution of the total dislocation density variation $\delta \rho(\boldsymbol{r})=\rho^{+}(\boldsymbol{r})+\rho^{-}(\boldsymbol{r})-\rho$ becomes

$$
\partial_{t} \delta \rho(\boldsymbol{r})=-b \mu \rho \nabla_{x} \int \delta \rho\left(\boldsymbol{r}^{\prime}\right) d_{\rho}\left(\boldsymbol{r}-\boldsymbol{r}^{\prime}\right) \sigma\left(\boldsymbol{r}-\boldsymbol{r}^{\prime}\right) d^{2} r^{\prime},
$$

where the effective pair correlation functions are those in the initial, homogeneous dislocation arrangement. We expand $\delta \rho\left(\boldsymbol{r}^{\prime}\right)$ up to fourth order in $r$. This yields

$$
\frac{1}{b \mu} \frac{\partial[\delta \rho]}{\partial t}=-D_{x}^{(2)} \frac{\partial^{2}[\delta \rho]}{\partial x^{2}}-D_{x x}^{(4)} \frac{\partial^{4}[\delta \rho]}{\partial x^{4}}-D_{x y}^{(4)} \frac{\partial^{4}[\delta \rho]}{\partial x^{2} \partial y^{2}}
$$

with the second-order expansion coefficient $D_{x}^{(2)}$ $=\int \tilde{x} d_{\rho}(\tilde{\boldsymbol{r}}) \sigma(\tilde{\boldsymbol{r}}) d^{2} \tilde{r}$, and the fourth-order expansion coefficients $\quad D_{x x}^{(4)}=[1 / \rho] \int\left(\tilde{x}^{3} / 6\right) d_{\rho}(\tilde{\boldsymbol{r}}) \sigma(\tilde{\boldsymbol{r}}) d^{2} \tilde{r} \quad$ and $\quad D_{x y}^{(4)}$ $=[1 / \rho] \int\left(\tilde{x} \tilde{y}^{2} / 2\right) d_{\rho}(\tilde{\boldsymbol{r}}) \sigma(\tilde{\boldsymbol{r}}) d^{2} \tilde{r}$. A numerical evaluation of these terms using the stress field of an edge dislocation and the pair correlation functions depicted in Figs. 3(a) and 3(b) yields $\quad D_{x}^{(2)}=2.02 \times 10^{-3} \mathrm{~Gb} /(1-\nu), D_{x x}^{(4)}=2.2710^{-3} \mathrm{~Gb} /$ $[\rho(1-\nu)], D_{x y}^{(4)}=9.24 \times 10^{-3} G b /[\rho(1-\nu)]$ where $\nu$ is Poisson's number. For all other derivatives of $\delta \rho$ of less than sixth order the corresponding coefficients vanish for symmetry reasons.

Inserting the ansatz $\delta \rho(\boldsymbol{r}, t)=\delta \rho_{0} \exp [\Lambda(\boldsymbol{q}) t] \exp ($ iqr $)$ into Eq. (20) yields the dispersion relation

$$
\Lambda(\boldsymbol{q})=b \nu\left[D_{x}^{(2)} q_{x}^{2}-D_{x x}^{(4)} q_{x}^{4}-D_{x y}^{(4)} q_{x}^{2} q_{y}^{2}\right]
$$

$\Lambda(\boldsymbol{q})$ can be interpreted as an "amplification factor" of the corresponding mode. The mode with maximum amplification has wave vector in the $x$ direction and wavelength $\lambda\left(\Lambda_{\max }\right)$ $=2 \pi \sqrt{2 D_{x}^{(4)} / D_{x}^{(2)}} \approx 9.42 / \sqrt{\rho}$. This indicates that the dislocation arrangement is unstable with respect to patterning on a length scale of about 10 average dislocation spacings. This is in qualitative agreement with observations reported in Ref. 34 which give $\lambda \sqrt{\rho} \approx 15$. The characteristic time for the growth of the mode with maximum amplification is given by $\Lambda_{\max }^{-1} \approx 2.2 \times 10^{3}(1-\nu) /\left[\rho G b^{2} \mu\right]$. This time is proportional to $1 / \rho$, in agreement with the scaling prediction of Sec. II B. We note that the characteristic time for establishing the quasi-steady-state pair correlation functions $d_{++}$and $d_{+-}$ out of an initially random dislocation arrangement is less by a factor of about 100, i.e., patterning proceeds on a slow time scale on which one can use a quasistatic approximation for the pair correlation functions. 


\section{DISCUSSION AND CONCLUSIONS}

In order to investigate the role of long-range dislocationdislocation interaction in dislocation pattern formation a system of straight parallel dislocation is considered. We have demonstrated that pair correlations in the dislocation arrangement introduce into the equations of evolution of the dislocation densities a length scale which is proportional to the average dislocation spacing. This result depends only on general properties of the evolution equations for the manydislocation densities (or correlation functions). Since these equations can be derived from the dynamics of the discrete dislocations in a rigorous manner, we have demonstrated for the first time how length scales in a density-based formulation of dislocation dynamics can be obtained without invoking ad hoc assumptions. To achieve this it was, however, necessary to go beyond single-dislocation densities and to take into account the presence of correlations in the dislocation arrangement in an explicit manner. It is expected that the present results carry over to more complicated deformation geometries: Because of the general scaling relations discussed in Sec. II, any pair correlation functions in a homogeneous and stationary dislocation arrangement depend on the scaled coordinate $\tilde{\boldsymbol{r}}=\boldsymbol{r} \sqrt{\rho}$ only, and therefore length scales of patterns derived from an analysis as in Sec. V will always be proportional to the average dislocation spacing. The pattern wavelength of the order of 10 average dislocation spacings which we derive is of the order of typical values observed by experiment.

What is the physical origin of this patterning? Our Eq. (21) is very similar to the equation $\mathrm{Holt}^{7}$ derived within an irreversible thermodynamics framework, although the formal approach we use is completely different: While the present results are derived from statistically averaging the dynamics of discrete dislocations, Holt's results derive from the assumption that the dynamics of dislocation densities (as opposed to individual dislocations) minimizes an energy functional, i.e., he considers "energetically driven" dislocationdensity patterning. Comparison of our results with those of Holt may be useful to achieve an improved conceptual understanding of the mechanisms governing the evolution of dislocation patterns.

As pointed out by Wilkens, ${ }^{8}$ without invoking pair correlations it is virtually impossible to formulate a meaningful energy functional which relates the stored elastic energy to the dislocation densities. Holt obtains a nonsingular energy functional by assuming a pair correlation function which is consistent with our Eq. (16). More generally speaking: because of the necessity of introducing pair correlations, any "energetic" theory which uses dislocation densities as state variables necessarily contains hidden variables, viz the pair correlation functions. The properties of these functions may be of crucial importance for the dynamics. Holt's main result, namely, a $\lambda \sim 1 / \sqrt{\rho}$ proportionality, relies on the (correct) ad hoc assumption that the range of dislocation pair correlations scales in proportion with the dislocation spacing. The correlation function is assumed isotropic, and the anisotropy of dislocation interactions and the constraints arising from dislocation motion on glide planes are neglected. Ac- cordingly the result is an isotropic $\Lambda(\boldsymbol{q})$ function. In the present work, on the other hand, we find that anisotropy of dislocation motion and interactions leads to anisotropic correlations (Figs. 1-3). Accordingly, patterning is strongly anisotropic. For the slip geometry analyzed in Sec. V, the mode of maximum instability characterizes density modulations in the slip direction while any density modulations normal to this direction are damped, i.e., the instability leads to the formation of "dislocation walls" normal to the slip plane.

In extreme cases, a "wrong" pair correlation function may even suppress the patterning. This is seen, for instance, by inserting into Eq. (19) the pair correlation function $\widetilde{d}(\tilde{\boldsymbol{r}})$ $=d_{0}(\tilde{r}) \delta(\theta-\pi / 2)$ where the radial function $d_{0}(\tilde{r})$ is chosen such that $\widetilde{d}(\tilde{\boldsymbol{r}})$ complies with the properties formulated in Sec. III. Evaluating Eq. (19) with this correlation function yields $\Lambda(\boldsymbol{q})=0$ for all $\boldsymbol{q}$. The reason is the following: Energetically driven dislocation-density patterning results from the fact that dislocations, by clustering, reduce their screening radius and thereby the elastic energy. For the fictitious pair correlation function $\widetilde{d}$, however, only a densification in the $y$ direction would enhance screening. This is impossible since, for the slip geometry considered, dislocations can move in the $x$ direction only. Our particular choice of the pair correlation function in this counterexample is, of course, completely unphysical- $\widetilde{d}$ corresponds to a random arrangement of pairs of edge dislocations of opposite signs where the dislocations of each pair are vertically above each other, i.e., in an unstable configuration. However, there is no $a$ priori method to decide which pair correlation function is physically correct. Apart from an educated guess, one has to rely on determination of pair correlations from discrete dislocation dynamics simulations or on solving the integrodifferential equations for the pair densities.

To summarize, we note that any dislocation patterning is "energetically driven" in the sense that the motion of dislocations is driven by forces, i.e., they move downhill in an energy functional. However, the question is how the highdimensional energy functional which characterizes the manydislocation system can be projected on a low-dimensional phase space where (single-)dislocation densities are the only state variables. We have shown that already the very definition of an elastic energy functional necessitates the consideration of pair correlations which, from the point of view of a density-based dynamics, are hidden variables. Since their properties influence the emerging patterns both quantitatively and qualitatively, these variables should be made visible and considered explicitly. In dynamic situations an even stronger caveat applies. In materials with high dislocation mobility, the dislocation system is during plastic deformation in a close-to-critical state. ${ }^{35,36}$ Under these circumstances, correlation functions of all orders become relevant and it is virtually impossible to project the high-dimensional dynamics of the interacting dislocations on a low-dimensional phase space. Models of dislocation motion and patterning in such situations must explicitly account for the high-dimensional nature of the dynamics. This can be done by introducing appropriately defined random processes into the evolution equations for the dislocation densities. ${ }^{9,35,36}$ The influence of 
these random processes depends on how they couple to the dislocation densities. (i) The "noise" due to collective dislocation motions may be neglected when it simply superimposes on a slow and low-dimensional, energetically driven pattern evolution. (ii) When the "noise" couples to the dislocation densities in a multiplicative manner, however, it is not possible to disentangle the fast, high-dimensional dynamics of the interacting dislocations from the slow dynamics of the patterns. In such situations, the method used in the present work (as well as any low-dimensional "energetic" approach) is not adequate for characterizing the evolution of dislocation structures.

\section{ACKNOWLEDGMENTS}

I.G. acknowledges financial support of the Hungarian Scientific Research Fund (OTKA) under Contract No. T 030791, and M.Z. support of the Commission of the European Communities under Contract No. ERB FMRXCT96-0062 and of the Deutsche Forschungsgemeinschaft.
${ }^{1}$ S.V. Raj and G.M. Pharr, Mater. Sci. Eng., 81, 217 (1986).

${ }^{2}$ M. Zaiser and P. Hähner, Mater. Sci. Eng., A 270, 299 (1999).

${ }^{3}$ D. Walgraef and E.C. Aifantis, Int. J. Eng. Sci. 23, 1351 (1985); 23, 1359 (1985); 23, 1365 (1985).

${ }^{4}$ D. Walgraef and E.C. Aifantis, J. Appl. Phys. 15, 688 (1985).

${ }^{5}$ J. Kratochvil, Scr. Metall. 20, 1625 (1986).

${ }^{6}$ J. Kratochvil, Scr. Metall. Mater. 26, 113 (1993).

${ }^{7}$ D.L. Holt, J. Appl. Phys. 41, 3197 (1970).

${ }^{8}$ M. Wilkens, Acta Metall. 15, 1412 (1967); 15, 1415 (1967); 17, 1155 (1969).

${ }^{9}$ P. Hähner, Appl. Phys. A: Mater. Sci. Process. 62, 473 (1996).

${ }^{10}$ P. Hähner, Acta Mater. 44, 2345 (1996).

${ }^{11}$ J. Gil Sevillano, Phys. Scr., T 49, 405 (1993).

${ }^{12}$ P. Hähner, K. Bay, and M. Zaiser, Phys. Rev. Lett. 81, 2470 (1998).

${ }^{13}$ M. Zaiser, K. Bay, and P. Hähner, Acta Mater. 47, 2463 (1999).

${ }^{14}$ F. Szekely, I. Groma, and J. Lendvai, Phys. Rev. B 62, 3093 (2000)

${ }^{15}$ M. Zaiser, Mater. Sci. Eng. A 249, 145 (1998).

${ }^{16}$ L.P. Kubin and G.R. Canova, Scr. Metall. Mater. 27, 957 (1992).

${ }^{17}$ N.M. Ghoniem, S.H. Tong, and L.Z. Sun, Phys. Rev. B 61, 913 (2000).

${ }^{18}$ B. Devincre and L.P. Kubin, Mater. Sci. Eng., A 234-236, 8 (1997).

${ }^{19}$ B. Devincre, L.P. Kubin, C. Lemarchand, and R. Madec, Mater. Sci. Eng., A 309-310, 211 (2001).

${ }^{20}$ I. Groma, Phys. Rev. B 56, 5807 (1997).

${ }^{21}$ A. El-Azab, Phys. Rev. B 61, 11956 (2000).

${ }^{22}$ E. Kröner, Kontinuumstheorie der Versetzungen und Eigenspannungen (Springer, Berlin, 1958).

${ }^{23}$ E. Kröner, in Physics of Defects, edited by R. Balian, M. Kleman, and J.-P. Poirier (North-Holland, Amsterdam, 1981), p. 219.

${ }^{24}$ E. Kröner, in Inelastic Behaviour of Solids, edited by M.F. Kan- ninen, W.F. Adler, A.R. Rosenberg, and R.I. Jaffee (McGrawHill, New York 1969), p. 137.

${ }^{25}$ Y.L. Klimontovich, Statistical Theory of Non-Equilibrium Processes in a Plasma (MIT Press, Cambridge, MA, 1967).

${ }^{26}$ I. Groma and P. Balogh, Acta Mater. 47, 3647 (1999).

${ }^{27}$ I. Groma and B. Bako, Phys. Rev. Lett. 84, 1487 (2000).

${ }^{28}$ M. Zaiser and A. Seeger, in Dislocations in Solids, edited by F.R.N. Nabarro (North-Holland, Amsterdam, in press).

${ }^{29}$ E. Kotomin and V. Kuzovkov, Modern Aspects of DiffusionControlled Reactions (Elsevier, Amsterdam 1996).

${ }^{30}$ J. Friedel, Dislocations (Pergamon, Oxford 1964).

${ }^{31}$ Under the conditions of typical deformation experiments, the assumption of quasistationary dislocation arrangements is well fulfilled since only a very small fraction of the dislocations in each moment actually moves (see Ref. 35). Deviations from similitude are, however, observed when dislocations acquire additional degrees of freedom, e.g., when cross-slip of screw dislocations sets in above a critical stress.

${ }^{32}$ Suppose we have a dislocation arrangement with this property. Adding further excess dislocations without violating Eq. (16) is possible if these form an arrangement which does not create shear stresses in the slip system $\alpha_{1}$. This requires that the additional excess dislocations must either have zero net Burgers vector or form infinitely extended dislocation walls which would, however, violate the boundary condition Eq. (12). A more detailed discussion of screening in dislocation arrangements will be given elsewhere.

${ }^{33}$ M.-C. Miguel, A. Vespignani, S. Zapperi, J. Weiss, and J.-R. Grasso, Mater. Sci. Eng., A 309-310, 324 (2001).

${ }^{34}$ M.R. Staker and D.L. Holt, Acta Metall. 20, 569 (1972).

${ }^{35}$ M. Zaiser, Mater. Sci. Eng., A 309-310, 304 (2001).

${ }^{36}$ M.-C. Miguel, A. Vespignani, S. Zapperi, J. Weiss, and J.-R. Grasso, Nature (London) 410, 667 (2001). 